\title{
MORTALIDAD Y FACTORES SOCIALES DEL CÁNCER DE PRÓSTATA PARA LA GESTIÓN DE POLIITICAS PÚBLICAS. VERACRUZ, MÉXICO
}

Magdoly Hernández Morales ${ }^{1}$, Ángel Patricio Hernández Nieves ${ }^{1}$, Sergio Arturo García Grajales ${ }^{2}$, Rosa Llisel Ocampo Escobedo ${ }^{2}$, Marco Antonio Luna Ruiz Esparza $^{2}$, Christian Soledad Ortiz Chacha ${ }^{3}$, Dulce María Cinta Loaiza ${ }^{3}$, Jaime Morales Romero ${ }^{3}$

\section{Resumen}

Introducción: las elevadas tasas de mortalidad por cáncer de próstata en Veracruz indican la necesidad de gestionar políticas públicas equitativas para su control. Por ello, es necesario analizar las tendencias de mortalidad y los factores sociales relacionados con la salud de la población. Métodos: cálculo de las tasas de mortalidad a nivel nacional y estatal por edad, y en Veracruz, por regiones económicas; en relación con su grado de rezago social. Resultados: La tasa de mortalidad por cáncer de próstata en Veracruz es mayor que la tasa nacional. Dentro del estado, la región del Papaloapan presentó la mayor mortalidad, y un grado de rezago social medio. Conclusiones: De acuerdo con los datos analizados, las regiones con las tasas más altas de mortalidad en Veracruz no corresponden a las regiones con alto grado de rezago social.

Palabras clave: cáncer de próstata, mortalidad, tasas de mortalidad, políticas públicas, equidad, salud.

${ }^{1}$ Alumnos de la maestría en Salud Pública, Instituto de Salud Pública, Universidad Veracruzana.

${ }^{2}$ Alumno de la especialidad en Epidemiología, IMSS, Instituto de Salud Pública, Universidad Veracruzana.

${ }^{3}$ Académicos del Instituto de Salud Pública, Universidad Veracruzana.

Correspondencia: Magdoly Hernández Morales. (hernandez_890@hotmail.com) Instituto de Salud Pública, Universidad Veracruzana. Av. Luis Castelazo Ayala s/n. Col. Industrial Ánimas. CP. 91190. Xalapa, Veracruz, México. 


\section{MORTALITY AND SOCIAL FACTORS OF PROSTATE CANCER FOR THE MANAGEMENT OF PUBLIC POLICIES. VERACRUZ MEXICO}

Magdoly Hernández Morales ${ }^{1}$, Ángel Patricio Hernández Nieves ${ }^{1}$, Sergio Arturo García Grajales², Rosa Llisel Ocampo Escobedo², Marco Antonio Luna Ruiz Esparza $^{2}$, Christian Soledad Ortiz Chacha ${ }^{3}$, Dulce María Cinta Loaiza ${ }^{3}$, Jaime Morales Romero ${ }^{3}$

\section{Abstract}

Introduction: The high rates of mortality caused by prostate cancer in Veracruz indicate the need to manage equitable public policies for its control. For this reason, it is necessary to analyze mortality trends and social factors related to population health. Methods: Calculation of mortality rates at national-state level, by age; and for Veracruz, by economic regions; in relation to its degree of social lag. Results: The mortality rate of prostate cancer in Veracruz is higher than the national rate. Within the state, the Papaloapan region presented the highest mortality, and an average degree of social lag. Conclusions: In Veracruz, according to the data analyzed, the regions with the highest mortality rates do not correspond to regions with a high degree of social lag. 


\section{MORTALIDADE E FATORES SOCIAIS DO CÂNCER DE PRÓSTATA PARA A GESTÃO DE POLIITICAS PÚBLICAS. VERACRUZ, MÉXICO}

Magdoly Hernández Morales ${ }^{1}$, Ángel Patricio Hernández Nieves ${ }^{1}$, Sergio Arturo García Grajales², Rosa Llisel Ocampo Escobedo ${ }^{2}$, Marco Antonio Luna Ruiz Esparza $^{2}$, Christian Soledad Ortiz Chacha ${ }^{3}$, Dulce María Cinta Loaiza ${ }^{3}$, Jaime Morales Romero ${ }^{3}$

\section{Resumo}

Introdução: As altas taxas de mortalidade por câncer de próstata em Veracruz indicam a necessidade de gerenciar políticas públicas equitativas para seu controle. Para isso, é necessário analisar tendências de mortalidade e fatores sociais relacionados à saúde da população. Métodos: Cálculo das taxas de mortalidade a nível nacional e estatal, por idade; e para Veracruz, por regiões econômicas; em relação ao seu grau de atraso social. Resultados: A taxa de mortalidade por câncer de próstata em Veracruz é superior à taxa nacional. Dentro do estado, a região de Papaloapan apresentou a maior mortalidade e um grau de atraso social médio. Conclusões: De acordo com os dados analisados, em Veracruz, as regiões com maiores taxas de mortalidade não correspondem a regiões com alto grau de atraso social. 


\section{Introducción}

Al hablar de equidad en salud, es posible concebir la integración de dos campos estrechamente relacionados: el ámbito de la salud integrado por dimensiones que involucran resultados finales de salud, el acceso a ella (no solo la disponibilidad de recursos o a la cobertura, sino la utilización efectiva de los servicios de salud, de acuerdo con los parámetros de necesidad) y la calidad de la asistencia sanitaria (1); y el ámbito social de la salud, conformado por los determinantes sociales, económicos, políticos y culturales que influyen en la posición social, la ocupación, los ingresos y el nivel de educación de los miembros de una población y en las posibilidades de conservar su salud $(2,3)$.

En este sentido, y de acuerdo con la Organización Mundial de la Salud, la equidad en salud implica que todos tengan una oportunidad justa para lograr plenamente su potencial en salud y que se evite, de ser posible, que alguna persona se encuentre en desventaja para lograrlo (4). Equidad significa considerar las necesidades individuales de las personas para propiciar el máximo nivel de salud posible (3).

Se sabe que los grupos de población en condición de pobreza son más propensos a enfermarse y afrontan mayores dificultades para acceder a servicios de salud de calidad. Estos colectivos registran tasas de mortalidad y morbilidad mayores en comparación con otros grupos de población, debido a la presencia de enfermedades no transmisibles como las cardiopatías, las enfermedades res- piratorias crónicas y el cáncer (5). Las desigualdades sociales en cuanto a la atención de cáncer en materia de prevención, detección, tratamiento y supervivencia (incluyendo calidad de vida después del tratamiento) tienen relación directa con las condiciones que posee cada individuo, es decir, cobertura y acceso a los servicios de salud, ocupación, ingresos económicos, nivel educativo, identidad sexual o de género, ubicación geográfica, entre otros (6). En general, cuando las condiciones sanitarias son desfavorables, la mortalidad por cáncer incrementa y la supervivencia y calidad de vida de los pacientes disminuye (7).

El cáncer se identifica como la segunda causa de muerte en el mundo; en 2015, ocasionó 8,8 millones de defunciones y casi una de cada seis defunciones en el mundo se debe a esta enfermedad. Cerca del 70\% de las muertes por cáncer se registran en países de ingresos medios y bajos (8). En las Américas, el cáncer es la segunda causa de muerte; se estima que 2,8 millones de personas son diagnosticadas cada año y 1,3 millones mueren (8). El cáncer se ha relacionado como resultado de ciertos estilos de vida y es, por tanto, considerado una consecuencia de las condiciones en las que las personas viven y trabajan (5).

Los tipos de cáncer más frecuentes en hombres son el de pulmón, próstata $y$ el colorrectal; en las mujeres, son de mama, pulmón y cáncer de cuello uterino (9). Entre estos tipos de cáncer destaca el de próstata, dado que en el 2012 se posicionó como la segunda neoplasia más incidente $(30,7$ por cada 100000$)$ a nivel mundial. En el mismo año, el cán- 
cer de próstata presentó la mayor tasa de incidencia en las Américas (75 por cada $100000)$, dejando en segundo lugar al cáncer de mama (10).

El cáncer de próstata se origina cuando las células de la próstata comienzan a crecer sin control (11). Los principales factores de riesgo de esta patología son: la edad, dado que la probabilidad aumenta después de los 50 años; los factores genéticos o hereditarios, es decir, si un padre o un hermano de un hombre padece cáncer de próstata, se duplica el riesgo de padecerla en el futuro (11); y la raza o grupo étnico, puesto que ocurre con más frecuencia en los hombres de raza negra y en hombres del Caribe con ascendencia africana. Otros factores con un efecto menos claro, pero que se han reportado para este tipo de cáncer son: alimentación, obesidad, exposición a sustancias químicas, inflamación de la próstata, infecciones de transmisión sexual y vasectomía (12).

Además de estos factores, expertos en el tema refieren que las altas tasas de mortalidad están íntimamente relacionadas con los factores económicos, ya que el costo para detectar, tratar y monitorear el cáncer de próstata es alto, tanto para el sistema de salud como para los individuos que lo padecen, y esto contribuye al empobrecimiento de las familias(13). En México, Márquez et al. (14) identificaron que el costo total en 2005 del tratamiento del cáncer de próstata fue de 1929004 pesos mexicanos, con un gasto promedio por paciente de \$17 697 (\$4 462 \$40 621, desviación estándar de 9929). También estimaron un gasto hospitalario promedio de \$24146, (mínimo de
\$3405, máximo de \$59000), equivalente al $0,01 \%$ del gasto del presupuesto hospitalario. De acuerdo con sus conclusiones, esto representa un gasto promedio de $111 \%$ del salario mensual nacional para la detección y control del cáncer de próstata, por lo que consideran al cáncer de próstata una enfermedad incapacitante económicamente en nuestro país.

En México, el cáncer de próstata ocupó el segundo lugar de incidencia (27,3 por cada 100000 ), colocándose solo por debajo del cáncer de mama. Sin embargo, el cáncer de próstata registró la tasa de mortalidad más alta (11,3 por cada 100 000) de todas las neoplasias malignas (10) mientras que para Veracruz en el mismo año se reportó una tasa de mortalidad de 15,43 por cada 100000 , superando la registrada a nivel nacional (15).

De acuerdo con un análisis de la mortalidad por cáncer de próstata reportada entre 1980 y 2013, realizado por investigadores del Instituto Nacional de Salud Pública (INSP) en México, los estados de Chiapas, Guerrero y Oaxaca (clasificados con muy alta marginación social), así como Campeche, Hidalgo, Michoacán, Puebla, San Luis Potosí, Tabasco, Veracruz y Yucatán (con alta marginación social) tuvieron las tasas de mortalidad más bajas o presentaron una reducción significativa de los casos de cáncer de próstata. A pesar de ello, el crecimiento en la mortalidad por este cáncer, observado en los últimos años de estudio, ocurrió principalmente en estos estados. Los autores refieren que estas tendencias se explican ante la relación dada por los niveles educativos, la dificultad de acceso a centros de salud 
y los factores culturales o creencias que obstaculizan la aceptación del diagnóstico y tratamiento del cáncer de próstata, especialmente en el grupo de hombres mayores de 65 años (16).

\section{¿Por qué es necesario gestionar políticas públicas equitativas para el control del cáncer de próstata?}

Los principales objetivos del control del cáncer de próstata son: reducir las tasas de mortalidad e incidencia; tener control sobre las causas y consecuencias; y mejorar la calidad de vida de los pacientes afectados y de sus familias. En México y Veracruz, la magnitud de este problema de salud pública es un indicador que resalta la necesidad de formular un programa para su control que aplique, de manera sistemática y equitativa, estrategias basadas en datos para la prevención, la detección temprana, el tratamiento y la paliación de la enfermedad. Asimismo, que haga un buen uso de los recursos disponibles, con una gestión adecuada que incluya procesos de planificación, aplicación, monitoreo y evaluación. Los programas de control del cáncer constituyen el medio más racional para lograr un grado de control de la enfermedad, aun contando con recursos limitados. (5)

Para dar respuesta a tal problemática en México, se crea el Programa de Acción para la Prevención y Control del Cáncer de Próstata (PAPCCP), integrado en el Programa Nacional de Salud 2001-2006 (11), como política pública para la atención del cáncer de próstata. Fue dirigido 168 a la población masculina mayor de 40 años y se enfocó a la prevención de este cáncer mediante acciones de promoción de la salud, detección y tratamiento oportuno de la enfermedad. Sin embargo, en el periodo del 2007 al 2012 no se encuentran registros de la continuidad o seguimiento a dicho programa. No obstante, durante este sexenio, en el año 2011 se incluyó el tratamiento para cáncer de próstata en el Fondo de Protección contra Gastos Catastróficos (17), el cual ofrece cobertura financiera de servicios médicos de alta especialidad a las personas que no cuentan con seguridad social y que padecen enfermedades de alto costo que pueden poner en riesgo su vida y patrimonio familiar.

En el Programa Sectorial de Salud 20132018 (18), dentro del objetivo "asegurar el acceso efectivo a servicios de salud con calidad" (p. 52) se encuentra la estrategia "mejorar el proceso para la detección y atención de neoplasias malignas, principalmente cáncer cérvico-uterino, de mama y próstata" con la línea de acción "fomentar la revisión rutinaria para detectar factores de riesgo de enfermedad prostática, cáncer de próstata y otras neoplasias malignas" ( $\mathrm{p}$. 53). A pesar de ello, solo se encuentran registros del Programa de Acción Específico de Prevención y Control del Cáncer de la Mujer 2013 - 2018 (19) lo que deja de lado el cáncer de próstata propio en hombres. Sin embargo, uno de los principios del Programa Sectorial de Salud es la equidad de género, la cual evidentemente no se contempla, pues solo cuenta con un programa para el cáncer de la mujer.

Con el fin de que el personal de salud que presta servicios de atención al cán- 
cer de próstata en las diferentes instituciones del Sistema Nacional de Salud siga procedimientos homogéneos al brindar atención, se reunió evidencia científica en un documento normativo. De este modo, en agosto del 2016, se publicó en el Diario Oficial de la Federación el Proyecto de Norma Oficial Mexicana PROY-NOM-048SSA2-2016 para la detección, diagnóstico, tratamiento y vigilancia epidemiológica del crecimiento prostático benigno (hiperplasia de la próstata) y de cáncer de próstata (tumor maligno de la próstata) (20). A través de este proyecto se fomenta la detección temprana para realizar un diagnóstico y tratamiento oportunos, acciones similares a las que se implementaron en el PAPCCP 2001-2006.

Entre el 2011 y el 2012, en Veracruz se elaboró el Programa de Prevención de la Hiperplasia Prostática y Tratamiento contra Cáncer de Próstata, que tuvo por objetivo disminuir la morbilidad de dicha patología (21). En el año 2013, el Sistema para el Desarrollo Integral de la Familia del estado de Veracruz (DIF Veracruz) planeó establecer un programa de detección oportuna de cáncer de próstata en los 212 municipios veracruzanos (22).

Por otra parte, el Programa de Acción Específico Envejecimiento 2016, contempló acciones para detectar y tratar hiperplasias benignas de próstata para evitar el cáncer, para lo cual se planteó la siguiente meta anual: detectar 293 237 hiperplasias benignas y tratar 330 hiperplasias benignas para evitar el cáncer (23). Como se observa, las políticas públicas para el control del cáncer de próstata en Veracruz se han enfocado a la prevención y detección oportuna de la enfermedad, dejando de lado las condiciones de vida los pacientes, así como otras de sus necesidades, como son el tratamiento y los cuidados paliativos, lo que contribuye a que se presenten diferencias en el estado de salud de los pacientes, y estas corresponden a inequidades en salud.

\section{Materiales y métodos}

Es necesario realizar un análisis de la carga del cáncer tanto a nivel nacional como estatal como uno de los pasos iniciales para la gestión de un programa de control del cáncer. Igualmente, es importante realizar las condiciones sociales que determinan el estado de salud de la población. Para esto, se describe el índice de rezago social, pues se sabe que las poblaciones más desfavorecidas tienen mayor riesgo de morir a causa de cáncer (5).

Para la descripción y comparación de la mortalidad por cáncer de próstata, se calcularon tasas de mortalidad ajustadas por edad mediante estandarización simple. Se consideraron solo aquellas muertes con causa básica de defunción tumor maligno de la próstata con clave CIE 10 C61X (clasificación internacional de enfermedades $10^{a}$ revisión) ocurridas entre el 2010 y el 2014. Se comparó la tasa de mortalidad nacional con las tasas de mortalidad de los estados y, en el caso de Veracruz, el análisis de la mortalidad se realizó con base en sus diez regiones económicas. La información de las defunciones se obtuvo a través de la plataforma cubos dinámicos de la Dirección 
General de Información en Salud de la Secretaría de Salud.

Para evaluar las condiciones sociales que determinan el estado de salud de la población, se utilizó el índice de rezago social, creado por el Consejo Nacional de Evaluación de la Política de Desarrollo Social (CONEVAL), que resume cuatro indicadores de carencia social: rezago educativo, acceso a los servicios de salud, acceso a los servicios básicos en la vivienda y la calidad y espacios en la vivienda. Su cálculo se basa en la técnica estadística de componentes principales, usando los datos obtenidos de la Encuesta Intercensal 2015. Los resultados del índice se presentan en cinco estratos: muy bajo, bajo, medio, alto y muy alto rezago social. Finalmente, se hace un cruce de las variables rezago social y mortalidad por cáncer de próstata a nivel nacional y estatal.

\section{Resultados}

De acuerdo con el Instituto Nacional de Estadística y Geografía (24), en el año 2015, en México, 10925657 hombres tenían una edad igual o mayor a los 50 años (18,8\% de la población). En el estado de Veracruz, la cifra fue de 831 342 hombres de 50 años de edad o más (21,2\% de la población); estos hombres presentan uno de los factores de riesgo más identificados para el desarrollo de cáncer de próstata.

Respecto a mortalidad por cáncer de próstata en México, entre el 2010 y el 2014 se registraron 29381 muertes clasificadas con la clave CIE 10 C61X, con un promedio de 5876 defunciones por año. El estado de Veracruz tuvo un total de 2329 para el mismo periodo, con un promedio de 466 defunciones por año.

En la Tabla 1 se presentan las tasas de defunción por cáncer de próstata de los estados de la república mexicana entre los años 2010 y 2014, que fueron ordenados de acuerdo al índice de rezago social reportado para el año 2015 por CONEVAL. Nayarit fue el estado con la tasa de defunción más alta, mientras que Quintana Roo fue el estado con la tasa más baja. Veracruz estuvo clasificado con un grado muy alto de rezago social; se clasificó dentro de los primeros diez estados con las tasas más altas de defunción por cáncer de próstata por arriba de la media nacional.

De acuerdo con Instituto Nacional de Estadística y Geografía (INEGI), el estado de Veracruz se conforma en 10 regiones económicas (Tabla 2); la región de las Montañas es donde se concentra el mayor número de municipios con grado de rezago social alto y muy alto, seguido de la Totonaca y la Huasteca baja. 
Tabla 1. Tasas de defunción por cáncer de próstata en México por entidad federativa, de acuerdo a grado de rezago social (de 2010 a 2014)*.

\begin{tabular}{|c|c|c|c|c|c|}
\hline $\begin{array}{l}\text { Clasificación estatal por } \\
\text { grado de rezago social }\end{array}$ & 2010 & 2011 & 2012 & 2013 & 2014 \\
\hline Nacional & 12,41 & 12,56 & 12,92 & 12,98 & 13,38 \\
\hline \multicolumn{6}{|l|}{ Muy alto } \\
\hline Chiapas & 10,37 & 11,04 & 13,46 & 13,42 & 13,49 \\
\hline Guerrero & 12,54 & 12,97 & 11,76 & 11,93 & 11,21 \\
\hline Oaxaca & 9,72 & 11,03 & 13,70 & 11,56 & 13,45 \\
\hline Veracruz & 14,47 & 14,14 & 15,43 & 15,87 & 15,80 \\
\hline \multicolumn{6}{|l|}{ Alto } \\
\hline Campeche & 13,61 & 10,63 & 12,47 & 9,97 & 10,63 \\
\hline Hidalgo & 12,84 & 10,76 & 10,48 & 10,86 & 13,51 \\
\hline Michoacán & 18,80 & 16,98 & 17,84 & 16,46 & 17,33 \\
\hline Puebla & 9,96 & 9,75 & 11,60 & 11,52 & 9,94 \\
\hline San Luis Potosí & 13,48 & 13,93 & 14,22 & 17,77 & 15,47 \\
\hline Yucatán & 11,29 & 9,35 & 10,07 & 10,52 & 10,73 \\
\hline \multicolumn{6}{|l|}{ Medio } \\
\hline Durango & 12,53 & 11,86 & 14,67 & 9,73 & 14,68 \\
\hline Guanajuato & 13,45 & 11,65 & 12,58 & 14,59 & 12,92 \\
\hline Nayarit & 16,84 & 19,60 & 22,23 & 23,06 & 22,18 \\
\hline Tabasco & 13,41 & 11,79 & 11,51 & 11,56 & 12,69 \\
\hline Tlaxcala & 7,85 & 8,10 & 11,18 & 10,97 & 8,29 \\
\hline \multicolumn{6}{|l|}{ Bajo } \\
\hline Baja California & 8,89 & 10,17 & 8,29 & 10,88 & 10,91 \\
\hline Baja California Sur & 11,97 & 7,95 & 9,08 & 10,47 & 11,12 \\
\hline Colima & 12,93 & 16,35 & 16,35 & 19,92 & 20,21 \\
\hline Chihuahua & 14,51 & 14,29 & 12,55 & 13,86 & 13,90 \\
\hline Jalisco & 16,80 & 17,97 & 17,31 & 17,66 & 16,94 \\
\hline Estado de México & 8,39 & 8,72 & 8,47 & 9,02 & 9,22 \\
\hline Morelos & 13,19 & 12,70 & 14,18 & 13,16 & 13,78 \\
\hline Querétaro & 10,18 & 9,12 & 10,83 & 7,43 & 9,64 \\
\hline Quintana Roo & 6,21 & 5,48 & 4,45 & 3,65 & 6,11 \\
\hline Sinaloa & 15,98 & 16,30 & 17,31 & 17,66 & 16,94 \\
\hline Sonora & 15,59 & 16,77 & 15,51 & 14,12 & 15,93 \\
\hline Tamaulipas & 12,73 & 11,78 & 12,41 & 10,41 & 13,32 \\
\hline Zacatecas & 16,27 & 15,22 & 17,42 & 17,73 & 21,18 \\
\hline \multicolumn{6}{|l|}{ Muy bajo } \\
\hline Aguascalientes & 9,97 & 13,65 & 16,34 & 13,11 & 13,48 \\
\hline
\end{tabular}


Continuación Tabla 1. Tasas de defunción por cáncer de próstata en México por entidad federativa, de acuerdo a grado de rezago social (de 2010 a 2014)*.

\begin{tabular}{l|l|l|l|l|l}
\hline $\begin{array}{l}\text { Clasificación estatal por } \\
\text { grado de rezago social }\end{array}$ & 2010 & 2011 & 2012 & 2013 & 2014 \\
\hline $\begin{array}{l}\text { Coahuila } \\
\text { Distrito Federal (Ciudad } \\
\text { de México) } \\
\text { Nuevo León }\end{array}$ \\
\hline
\end{tabular}

Fuente: elaboración propia a partir del índice de marginación 2015, CONEVAL.

*Tasas por cada 100000 habitantes hombres de 10 años en adelante

De acuerdo con Instituto Nacional de Estadística y Geografía (INEGI), el estado de Veracruz se conforma en 10 regiones económicas (Tabla 2); la región de las Montañas es donde se concentra el mayor número de municipios con grado de rezago social alto y muy alto, seguido de la Totonaca y la Huasteca baja.

Tabla 2. Grado de rezago social en Veracruz por regiones económicas, 2015

\begin{tabular}{lr|r|r|r|r|r}
\hline \multicolumn{1}{c|}{ Región } & $\begin{array}{c}\text { Total de } \\
\text { municipios }\end{array}$ & Muy bajo & Bajo & Medio & Alto & Muy alto \\
\hline Huasteca alta & 10 & 0 & 3 & 1 & 6 & 0 \\
Huasteca baja & 23 & 1 & 2 & 7 & 9 & 4 \\
Totonaca & 15 & 1 & 2 & 2 & 8 & 2 \\
Nautla & 10 & 0 & 5 & 5 & 1 & 0 \\
Capital & 33 & 2 & 10 & 13 & 8 & 0 \\
Sotavento & 12 & 4 & 6 & 2 & 0 & 0 \\
Montañas & 57 & 2 & 9 & 17 & 19 & 10 \\
Papaloapan & 21 & 0 & 8 & 13 & 1 & 0 \\
Los Tuxtlas & 25 & 0 & 0 & 3 & 1 & 0 \\
Olmeca & 25 & 2 & 7 & 12 & 4 & 0 \\
Estatal & 212 & 12 & 52 & 75 & 57 & 16 \\
\hline
\end{tabular}

Fuente: índice de marginación del Consejo Nacional de Población, y regiones económicas, Instituto Nacional de Estadística y Geografía (INEGI).

La mortalidad por cáncer de próstata en el estado de Veracruz presentó variaciones según las regiones económicas en que las se divide (Tabla 3). En el periodo comprendido entre el 2010 y el 2014, la región del Papaloapan presentó la tasa más alta de defunción por este tipo cáncer de todo el estado de Veracruz, mientras que la región con la menor tasa de mortalidad fue la región de las Montañas. 
Tabla 3. Tasas de defunción por cáncer de próstata en Veracruz por regiones (de 2010 a 2014).

\begin{tabular}{l|c|c|c|c|c|c}
\hline \multicolumn{1}{c|}{ Región } & $\mathbf{2 0 1 0}$ & $\mathbf{2 0 1 1}$ & $\mathbf{2 0 1 2}$ & $\mathbf{2 0 1 3}$ & $\mathbf{2 0 1 4}$ & $\mathbf{2 0 1 0 - 2 0 1 4}^{*}$ \\
\hline I Huasteca Alta & 11,10 & 14,87 & 14,13 & 17,85 & 12,03 & 14,00 \\
II Huasteca Baja & 17,66 & 13,98 & 14,65 & 17,99 & 16,72 & 16,20 \\
III Totonaca & 16,19 & 10,02 & 18,37 & 16,74 & 12,26 & 14,71 \\
IV Nautla & 15,91 & 12,97 & 18,94 & 14,07 & 17,93 & 15,96 \\
V Capital & 13,39 & 11,79 & 14,89 & 12,31 & 15,14 & 13,51 \\
VI Sotavento & 18,55 & 20,04 & 18,57 & 19,38 & 18,96 & 19,10 \\
VII Montañas & 9,95 & 9,59 & 12,00 & 11,31 & 10,46 & 10,66 \\
VIII Papaloapan & 17,87 & 23,32 & 23,07 & 21,55 & 21,36 & 21,44 \\
IX Los Tuxtlas & 20,42 & 21,82 & 7,47 & 15,61 & 19,52 & 16,97 \\
X Olmeca & 12,08 & 12,89 & 13,73 & 17,19 & 18,39 & 14,86 \\
Estatal & 14,47 & 14,14 & 15,43 & 15,87 & 15,80 & 15,14 \\
Nacional & 12,41 & 12,56 & 12,92 & 12,98 & 13,38 & 12,85 \\
\hline
\end{tabular}

Fuente: elaboración propia con datos de la SSA .

*Tasas por cada 100000 habitantes hombres de 10 años en adelante

${ }^{* *}$ Media de las tasas de defunción de los años 2010 al 2014

En el análisis por edad se observó una nal a la edad. Se observó de igual fortendencia de incremento de la tasa de ma a nivel nacional, estatal y regional mortalidad directamente proporcio- (Tabla 4).

Tabla 4. Tasas de defunción por quinquenios de vida en el estado de Veracruz.

\begin{tabular}{|c|c|c|c|c|c|c|}
\hline Edad & 2010 & 2011 & 2012 & 2013 & 2014 & 2010-2014* \\
\hline \multicolumn{7}{|l|}{10 a 14} \\
\hline \multicolumn{7}{|l|}{15 a 19} \\
\hline \multicolumn{7}{|l|}{20 a 24} \\
\hline 25 a 29 & & & 0,35 & & & 0,07 \\
\hline \multicolumn{7}{|l|}{30 а 34} \\
\hline 35 а 39 & & & & & 0,80 & 0,16 \\
\hline \multicolumn{7}{|l|}{40 a 44} \\
\hline 45 a 49 & 0,98 & 1,43 & 1,40 & 0,91 & 0,90 & 1,12 \\
\hline 50 a 54 & 2,84 & 1,11 & 3,80 & 3,71 & 3,11 & 2,91 \\
\hline 55 a 59 & 12,39 & 11,34 & 9,72 & 6,93 & 7,98 & 9,67 \\
\hline 60 a 64 & 23,46 & 21,09 & 24,53 & 27,71 & 21,46 & 23,65 \\
\hline
\end{tabular}


INVESTIGACIONES ANDINA No. 35, Vol. 19

Continuación Tabla 4. Tasas de defunción por quinquenios de vida en el estado de Veracruz.

\begin{tabular}{l|r|r|r|r|r|r}
\hline \multicolumn{1}{c|}{ Edad } & \multicolumn{1}{c|}{$\mathbf{2 0 1 0}$} & \multicolumn{1}{c|}{$\mathbf{2 0 1 1}$} & \multicolumn{1}{c|}{$\mathbf{2 0 1 2}$} & $\mathbf{2 0 1 3}$ & $\mathbf{2 0 1 4}$ & $\mathbf{2 0 1 0 - 2 0 1 4}$ \\
\hline 65 a 69 & 54,03 & 38,77 & 41,01 & 42,04 & 47,92 & 44,75 \\
70 a 74 & 108,65 & 95,03 & 98,50 & 97,66 & 108,72 & 101,71 \\
75 a 79 & 146,84 & 181,25 & 173,46 & 177,51 & 151,46 & 166,11 \\
80 a 84 & 284,04 & 279,95 & 291,85 & 336,70 & 305,83 & 299,67 \\
85 y más & 418,06 & 408,69 & 495,98 & 455,23 & 474,41 & 450,47 \\
Total & 14,44 & 14,07 & 15,43 & 15,84 & 15,80 & 15,12 \\
\hline
\end{tabular}

Fuente: elaboración propia con datos de la Secretaría de Salud del Estado de Veracruz. México.

* Casillas en blanco por ausencia de defunciones en esos quinquenios de edad.

${ }^{* *}$ Media de las tasas de defunción de los años 2010 al 2014.

*** Tasas por cada 100000 habitantes hombres de 10 y más años

El municipio con la mayor mortalidad por cáncer de próstata fue Tlacoltalpan, el cual pertenece a la región del Papaloapan. Tuvo una tasa de 55 defunciones por cada 100000 habitantes hombres de 10 años en adelante, muy por arriba de la media estatal $(15,14)$ y aún más arriba de la nacional $(12,85)$. En la Tabla 5 se muestran los municipios con mayor mortalidad por cáncer de próstata del estado de Veracruz.

Tabla 5. Primeros 10 municipios de Veracruz con mayor mortalidad por cáncer de próstata (de 2010 a 2014).

\begin{tabular}{lr|l|r|r|r|r|r|r}
\hline Región & Clave & Municipio & $\mathbf{2 0 1 0}$ & $\mathbf{2 0 1 1}$ & $\mathbf{2 0 1 2}$ & $\mathbf{2 0 1 3}$ & $\mathbf{2 0 1 4}$ & $\mathbf{2 0 1 0 - 2 0 1 4}$ \\
\hline Papaloapan & 178 & Tlacotalpan & 72,94 & 73,22 & 18,38 & 73,68 & 36,87 & 55,02 \\
Huasteca baja & 151 & Tamiahua & 49,99 & 9,98 & 29,96 & 29,94 & 99,66 & 43,91 \\
Papaloapan & 12 & Amatitlán & 64,66 & 96,12 & 0,00 & 31,72 & 0,00 & 38,50 \\
Sotavento & 90 & Jamapa & 71,16 & 23,43 & 0,00 & 23,04 & 68,59 & 37,24 \\
Sotavento & 181 & Tlalixcoyan & 19,67 & 19,43 & 38,53 & 51,00 & 57,00 & 37,13 \\
Sotavento & 49 & Cotaxtla & 37,34 & 36,82 & 72,90 & 12,04 & 23,89 & 36,60 \\
Papaloapan & 11 & Alvarado & 23,34 & 27,66 & 31,99 & 40,81 & 58,52 & 36,47 \\
Capital & 1 & Acajete & 0,00 & 59,86 & 88,76 & 0,00 & 28,87 & 35,50 \\
Nautla & 42 & Colipa & 44,01 & 43,82 & 0,00 & 43,50 & 43,29 & 34,92 \\
Huasteca baja & 150 & Tamalín & 0,00 & 85,74 & 42,74 & 42,58 & 0,00 & 34,21
\end{tabular}




\section{Discusión}

Dentro del estado de Veracruz existe polaridad entre sus regiones económicas, pues en la región de las Montañas casi la mitad de los municipios registraron un alto y muy alto grado de marginación. Sin embargo, es la región que presentó la tasa de mortalidad más baja para el periodo entre 2010 a 2014. Los municipios de la región del Papaloapan se clasificaron con índices de rezago social medio y bajo; no obstante, en el periodo 2010-2014 se observó la tasa de mortalidad más alta por cáncer de próstata de todo el estado de Veracruz, a diferencia de lo encontrado en Cali, Colombia, donde se identificó que los hombres con estrato socioeconómico bajo tenían mucha mayor probabilidad de morir en comparación con los hombres de estrato socioeconómico alto (5).

Del mismo modo, resultados similares se concluyeron en un estudio realizado por el INSP de México, pues la mortalidad por cáncer de próstata mostró el mayor incremento anual entre los estados con tasas de marginación muy altas (16). En Ginebra, Suiza, se encontró que los pacientes con cáncer de próstata de bajo nivel socioeconómico tenían un riesgo dos veces mayor de muerte por su enfermedad, en comparación con los pacientes con cáncer de próstata de alto nivel socioeconómico (26). De acuerdo con los datos analizados las regiones en Veracruz con las tasas más altas de mortalidad no corresponden a las regiones con alto grado de rezago social.

En el análisis por edad se observa la clara tendencia de incremento de la tasa de mortalidad conforme aumenta la edad, pues es en el grupo de 85 años y más, donde la tasa de mortalidad es mayor. Esta información concuerda con lo referido en las estimaciones del proyecto GLOBOCAN 2012, ya que el pico máximo de aparición de la enfermedad es después de los 65 años (10).

El acceso al diagnóstico y el tratamiento oportuno condicionan la dinámica de las defunciones por cáncer de próstata. Si bien es cierto que en el año 2011 se incluyó el tratamiento para cáncer de próstata en el Fondo de Protección contra Gastos Catastróficos para población sin seguridad social, en el estado de Veracruz únicamente existen dos hospitales certificados para dar atención de este padecimiento: el Centro Estatal de Cancerología "Dr. Miguel Dorantes Mesa", ubicado en la ciudad de Xalapa, y el Hospital de Alta Especialidad de Veracruz, ubicado en la ciudad de Veracruz. Esto significa que la región capital es una de las que reporta las tasas de mortalidad más baja, dado que los hospitales están ubicados en la región Capital y en Sotavento, respectivamente.

Esta distribución de infraestructura refleja la inequidad de acceso para la población veracruzana. Por ejemplo, el municipio Pánuco, ubicado en la región Huasteca Alta, se encuentra a 478,8 km de la ciudad de Veracruz y a 507,6 km de distancia de la ciudad de Xalapa; sus habitantes sin seguridad social tendrían que viajar aproximadamente 8 horas para aspirar a ser atendidos en uno de los dos hospitales certificados. También existe el caso del municipio de Las Choapas, ubicado en la región Olmeca. 
Este se encuentra a $353,1 \mathrm{~km}$ de Veracruz y a 459,3 km de Xalapa, así que el tiempo de traslado al hospital desde su localidad oscila entre 7 horas aproximadamente.

Si no existe un programa de acción a nivel nacional específico, equitativo y vigente que esté enfocado en la detección temprana, tratamiento y paliación para cáncer de próstata, es mucho más complicado que los estados implementen un programa que atienda esta neoplasia maligna. Por esta razón, es posible que otros programas tiendan a incluir solamente acciones o metas que no contemplen la magnitud de la problemática. Esto sucedió con el Programa de Acción Específico de Envejecimiento 2016, pues se plantearon metas anuales incongruentes (detectar 293237 hiperplasias benignas y tratar 330 hiperplasias benignas). Si se cumplieran dichas metas, únicamente se le daría tratamiento a un $0,11 \%$ de las hiperplasias detectadas.

La pregunta es: ¿qué pasa con los demás?, ¿en qué momento se atienden?; es cierto que las detecciones están pensadas como una forma preventiva del cáncer de próstata, pero si la hiperplasia no se trata después de detectarse, realmente no se está previniendo el cáncer de próstata. En este sentido, las acciones que se planean se están quedando cortas ante la magnitud del problema, ya que las mayoría de acciones que se realizan son campañas para la detección de hiperplasia prostática que no especifican qué acciones se toman tras estas detecciones.

176 Las políticas públicas no le están dando la importancia que se requiere a este pa- decimiento. Si bien es cierto que entre el año 2001 y el año 2006 se implementó un programa de acción específico para cáncer de próstata por primera vez a nivel nacional, en los siguientes sexenios no se le dio continuidad a nivel federal y actualmente no existe un programa de acción específico para este padecimiento, a pesar de que el Programa Sectorial de Salud 2013-2018 establece la equidad de género entre sus principios.

La situación es similar en el estado de Veracruz, debido a que, actualmente, no existe ningún programa específico para cáncer de próstata. En el Programa de Acción Específico de Envejecimiento 2016 se incluyeron acciones para detectar las hiperplasias benignas de próstata para evitar el cáncer, pero no se determina con claridad qué se hará después de dichas de detecciones; aunque estas acciones estén pensadas en prevención, si no se le da seguimiento, las tasas de mortalidad seguirán en aumento.

La ausencia de un programa para la atención de cáncer de próstata contribuye al aumento de las tasas de mortalidad de tal padecimiento, ya que éstas guardan relación directa con el acceso a los servicios de salud y la cobertura que se ofrece; el costo para detectar, tratar y monitorear el cáncer de próstata es muy alto, tanto que contribuye así al empobrecimiento de las familias.

Toda política pública debe respaldarse en la normatividad jurídica del país. Afortunadamente, en México ya se estableció el PROY-NOM-048SSA2-2016 para la detección, diagnóstico, tratamiento y vigilancia epidemiológica del 
crecimiento prostático benigno y de cáncer de próstata, indispensable para garantizar su legitimidad. Esta política estaría planteada en un programa específico y equitativo para cáncer de próstata que tenga un soporte burocrático y que involucre, además del sector salud, al sector educativo y económico, de modo que estas instancias sean las responsables de aplicar la política y velar por su desarrollo. Para asegurar que la

\section{Referencias}

1. Linares-Pérez $N$, López-Arellano $O$. Inequidades en la salud en México. Gaceta Médica de México. 2012;148(6):591-7

2. Ministerio de Sanidad, Consumo y Bienestar Social del Gobierno de España. [Internet]. Equidad en salud y desigualdades sociales en salud. S.F. [Citado 10 de julio 2017]. Disponible en: https://www.msssi.gob.es/profesionales/ saludPublica/prevPromocion/promocion/ desigualdadSalud/EquidadSaludyDSS. htm

3. Linares-Pérez N, López-Arellano O. La equidad en salud: propuestas conceptuales, aspectos críticos y perspectivas desde el campo de la salud colectiva. Medicina Social. 2008;3(3):247-59.

4. Organización Mundial de la Salud. [Internet]. Comisión sobre determinantes sociales de la salud: conceptos clave. S.F. [Citado 2017 julio 6]. Disponible en: http:// www.who.int/social_determinants/final_ report/key_concepts/es/

5. Organización Mundial de la Salud. Programas nacionales de control de cáncer: política pública se cumpla exitosamente no bastan la normatividad o mecanismos administrativos, es ineludible que ésta se dé a conocer, y que sea aceptada y adoptada por la población. Tal propósito puede lograrse con la educación, la propaganda (debe sujetarse a los principios jurídicos y éticos del país, en particular a la veracidad, la oportunidad, la imparcialidad y el respeto a las personas) y la publicidad. políticas y pautas para la gestión. Washington, D.C: Organización Panamericana de la Salud; 2004.

6. Arias S. Inequidad y cáncer: una revisión conceptual. Rev. Fac. Nac. Salud Pública. 2009;27(3):341-48.

7. Instituto Nacional de Cáncer. [Internet]. Estados Unidos: Institutos Nacionales de la Salud. Desigualdades por cáncer; 2018 marzo 29 [citado 2017 junio 15]. Disponible en: https://www.cancer.gov/espanol/ cancer/naturaleza/desigualdades

8. Organización Mundial de la Salud. [Internet]. Cáncer; 2018 febrero 1. [citado 2017 junio 12]. Disponible en: http://www. who.int/mediacentre/factsheets/fs297/es/

9. Organización Panamericana de la Salud. [Internet]. Las políticas públicas destinadas a favorecer estilos de vida saludables pueden prevenir más de un tercio de los cánceres; 2016 enero 29. [citado 2017 junio 30]. Disponible en: http://www.paho. org/hq/index.php?option=com_content\&view=article\&id $=11632 \% 3$ A2016-paho-urges-governments-to-implement-public-policies-that-can-prevent-cancer\&Itemid=1926\&lang=es 
10. International Agency for Research on Cancer/ World Health Organization. [Internet]GLOBOCAN 2012: Estimated Cancer Incidence, Mortality and Prevalence Worldwide in 2012; S.F. [citado 2016 noviembre 27]. Disponible en: http://globocan.iarc.fr/Pages/fact_sheets_population. aspx

11. Secretaría de Salud. Programa de acción: Cáncer de Próstata. [Internet]. México: Secretaría de Salud; 2001 [citado 2016 Diciembre 24]. 54 p. Disponible en: http://www.salud.gob.mx/unidades/cdi/documentos/cancer_prostata.pdf

12. American Cancer Society. [Online]. Factores de riesgo del cáncer de próstata.; 2016 abril 20 [citado 2016 noviembre 17]. Disponible en: https://www.cancer. org/es/cancer/cancer-de-prostata/causas-riesgos-prevencion/factores-de-riesgo.html

13. Ministerio de la Salud. Plan de acciones estratégicas para el enfrentamiento de las enfermedades no transmisibles (ent) en Brasil 2011-2022 [Internet]. Brasilia: Ministerio de la Salud; 2011. [citado 2016 diciembre 24]. Disponible en: http:// www.iccp-portal.org/sites/default/files/ plans/cartilha_13102011.pdf

14. Márquez J, et al. El costo del cáncer de próstata. ¿Qué esperamos? Rev Mex Urol. 2005;65(5):307-11.

15. Secretaría de Salud. [Internet]. Cubos dinámicos-Defunciones (mortalidad); 2018 febrero 6 [citado 2016 diciembre 24]. Disponible en: http://www.dgis.salud.gob. $\mathrm{mx} /$ contenidos/basesdedatos/bdc_defunciones_gobmx.html.

16. Torres-Sánchez L, et al.Prostate Cancer Mortality according to marginalization status in Mexican States from 1980 to 2013. Salud Pública Mex.2016;58(2):17986.

17. Secretaría de Salud. Intervenciones del fondo de protección contra gastos catastróficos. [Internet]. México: Secretaría de Salud; 2016 [citado 2016 diciembre 28]. 7 p. Disponible en: http://www.seguropopulartlax.gob.mx/intervencionesGastosCatastroficos.pdf

18. Gobierno de la República. Plan Nacional de Desarrollo, Programa Sectorial de Salud. [Internet]. México; 2013 [citado 2016 Diciembre 15]. 138 p. Disponible en: http://portal.salud.gob. $\mathrm{mx} /$ contenidos/conoce_salud/prosesa/ prosesa.html

19. Secretaría de Salud. Programa de Acción Específico Prevención y Control del Cáncer de la Mujer 2013-2018. [Internet]. México: Secretaría de Salud; 2013 [citado 2017 enero 5].Disponible en: http:// cnegsr.salud.gob.mx/contenidos/descargas/cama/PrevencionyControldelCancerdelaMujer_2013_2018.pdf

20. Diario Oficial de la Federación. PROYECTO de Norma Oficial Mexicana PROY-NOM-048-SSA2-2016, Para la detección, diagnóstico, tratamiento y vigilancia epidemiológica del crecimiento prostático benigno (hiperplasia de la próstata) y cáncer de próstata (tumor maligno de la próstata). [Internet]. Secretaría de Gobernación; 2016 [citado 2016 diciembre 26]. Disponible en: http://www.dof.gob.mx/ nota_detalle.php?codigo $=5446705 \&$ fecha $=\overline{0} 3 / 08 / 2016$

21. Inventario CONEVAL de programas y acciones Estatales de Desarrollo Social. [Internet]. México: Consejo Nacional de Evaluación de Política de Desarrollo Social (CONEVAL). Inventario 2011; 2016 [citado 2016 Octubre 25]. Disponible en: http://www.coneval.org.mx/Evaluacion/ IPE/Paginas/historico.aspx

22. Desarrollo Integral de la Familia. DIF Estatal de Veracruz. [Online]; 2012 [cited 2016 Noviembre 05]. Available from: http:// www.veracruzenlanoticia.com/2012/09/ erradicar-el-cancer-de-prostata-prioridad-del-dif-estatal/

23. Escobar A, García A. Programa de Acción Específico Envejecimiento 2016. 
[Internet]. Veracruz: Secretaría de Salud del Estado de Veracruz; 2016. [citado 2016 Octubre 28]. 39 p. Disponible en: https://www.ssaver.gob.mx/cronicasnotransmisibles/files/2016/09/PROGRAMA-ANUAL-DE-ENVEJECIMIENTO-2016-FINAL.pdf

24. Restrepo J, et al. Incidencia, mortalidad y supervivencia al cáncer de próstata en Cali, Colombia, 1962-2011. Salud Pública Mex. 2014;56(5):440-47

25. Rapiti E, et al. Impact of socioeconomic status on prostate cancer diagnosis, treatment, and prognosis. Cancer. [Internet]. 2009 [citado 2016 Diciembre 08]; 115(23):5556-65. Disponible en: http://onlinelibrary.wiley.com/doi/10.1002/ cncr.24607/pdf 
INVESTIGACIONES ANDINA No. 35, Vol. 19 


\section{Información}

\section{INSTRUCCIÓN A LOS AUTORES PARA LA PRESENTACIÓN DE ARTICULOS EN ESTA REVISTA}

A continuación se presentan la forma y preparación de los artículos para ser publicados en la revista.

\section{TÍTULO}

\section{AUTOR(ES)}

El orden de mención debe reflejar la magnitud e importancia de la contribución de cada individuo.

\section{INSTITUCIÓN(ES) Y DIRECCIÓN POSTAL}

Debe ir al pie de la primera página.

\section{RESUMEN}

El resumen debe ser estructurado así: Introducción, Métodos, Resultados y Conclusión.

El resumen debe ser máximo de 150 palabras. Los verbos usados se conjugan en tiempo pasado.

\section{PALABRAS CLAVE}

Facilitan las entradas (descriptores) en los sistemas de indización y de recuperación de la información. Por favor apóyese en los tesauros o en el www.bireme. br donde puede consultar el descriptor $\mathrm{DeC} / \mathrm{MeSH}$ y desde allí se le orientará para la definición de las palabras clave y también identificará la traducción de los términos de búsqueda.

\section{INTRODUCCIÓN}

En su elaboración se sugiere mencionar brevemente los siguientes aspectos:

1. El problema o el objetivo, indicando su origen, antecedentes e importancia.

2. Los conocimientos existentes sobre el tema, resumiendo los resultados de las investigaciones anteriores y su referencia bibliográfica.

3. Tipo de diseño utilizado, con una breve sustentación sobre su elección.

4. Definición de las principales variables, términos y abreviaturas.

5. Expresar el tiempo en presente. Un error es la ausencia de respaldo bibliográfico.

\section{MATERIALES Y MÉTODOS}

Detalla la manera como fueron obtenidos los resultados en cuanto al diseño, técnicas y materiales utilizados, para que la investigación pueda replicarse.

EN LOS MATERIALES NO SE OLVIDE INCLUIR

1. Especificaciones técnicas y cantidades exactas.

2. Nombre genérico, químico y comercial.

3. Procedencia, nombre del fabricante.

4. Técnicas de preparación. 
1. En caso de trabajar con animales, plantas y microorganismos, identificar género, especie $y$ cepa; características especiales de edad, sexo, condición genética y fisiológica.

2. En el caso de humanos, identificar las características principales de la población y de la muestra.

\section{EN LOS MÉTODOS PROCURE}

1. Expresar el tipo de diseño empleado.

2. Tipo de muestreo utilizado.

3. Mencionar las técnicas estadísticas y de los instrumentos de recolección de información; reportar indicadores de validez interna y externa de las pruebas empleadas sin recurrir a demostraciones; en caso de pertinencia, expresar la referencia bibliográfica.

4. Mencionar aspectos importantes del trabajo de campo.

5. Utilizar los verbos en tiempo pasado.

\section{PARA EL DISEÑO DEL ESTUDIO}

Es importante aclarar cómo fue la selección de sujetos u objetos y la asignación de sujetos u objetos a grupos de estudio (experimental, control, intervención tratamiento). Se debe ser específico acerca de la medición del efecto y de la exposición. Los métodos de análisis deben ser sustentados y acordes a los objetivos del estudio.

\section{RESULTADOS}

Esta parte del artículo tiene por objeto presentar la verdadera contribución del estudio realizado. En esta sección se presenta información pertinente a los objetivos del estudio en forma coherente y además las pruebas estadísticas aplicadas a la información recolectada. No olvide que debe mencionar todos los hallazgos relevantes, incluso aquellos contrarios a la hipótesis, también debe incluir detalles suficientes para justificar las conclusiones.

\section{PRESENTACIÓN DE LOS} RESULTADOS

Aquí el autor(es) puede(n):

1. Utilizar cuadros cuando sea necesario, resumir la información.

2. Cuando se trate de uno o pocos datos, descríbalos en el texto.

3. Las figuras estadísticas, croquis y esquemas ayudan a aclarar los resultados.

4. No debe repetir con palabras la información contenida en los cuadros y figuras, solamente expresar las conclusiones que se deduzcan de ellas.

5. Los datos estadísticos encontrados con pruebas de significancia, deben expresarse con interpretaciones y conclusiones claras, sencillas y concretas.

6. Los verbos en esta sección se recomienda que sean conjugados en tiempo pasado.

\section{REQUISITOS PARA CUADROS Y FIGURAS}

Los títulos de los cuadros van en la parte superior y los de las figuras se escriben en la parte inferior de las mismas.

1. Deben ser autoexplicativos, sencillos y de fácil comprensión.

2. Indicar el lugar, fecha y fuente de origen de la información. 
1. Se deben incluir unidades de medidas relativas (razones, porcentajes, tasas, índices).

\section{DISCUSIÓN}

$\mathrm{Su}$ fin es mostrar las interpretaciones, generalizaciones, relaciones, conclusiones y recomendaciones que los resultados indican y las discrepancias con otros hallazgos encontrados en investigaciones citadas en los Antecedentes y el Marco Teórico.

\section{SUGERENCIAS}

1. Exponga los principios, relaciones y generalizaciones que los resultados indican.

2. Señale las excepciones, la falta de correlación y delimite los aspectos no resueltos.

3. Muestre cómo concuerdan (o no) sus resultados e interpretaciones, con los trabajos anteriormente publicados.

4. Formule las conclusiones de la manera más clara posible.

5. Resuma las pruebas que respaldan cada conclusión.

6. Tiempo pasado para sus conclusiones y presente para las referencias bibliográficas. Las conclusiones son parte de la discusión de resultados y permiten inferir o deducir una verdad de otras que se admiten, demuestran o presuponen.

\section{AGRADECIMIENTOS}

Esta sección reconoce la cooperación de personas e instituciones que ayuda- ron materialmente al autor en su investigación.

\section{REFERENCIAS}

Las referencias permiten identificar las fuentes originales; dan solidez a los hechos y opiniones expresadas por el autor. Orientan además al lector para que se informe en mayor extensión. Para ampliar esta información, se le sugiere consultar las Normas Vancouver.

\section{REFERENCIAS PARA UNA REVISTA}

1. Autor(es) apellidos.

2. Título del artículo.

3. Nombre de la revista.

4. Año de la publicación.

5. Número del volumen.

6. Página (primera y última).

\section{REFERENCIAS PARA UN LIBRO}

1. Autor(es) o Editor(es).

2. Título.

3. Edición (si no es la primera).

4. Ciudad, sede de la editorial.

5. Editorial.

6. Año de la publicación.

\section{APÉNDICES O ANEXOS}

Para completar o ilustrar el desarrollo del tema, se debe incluir información que por su extensión o configuración no encuadra bien dentro del cuerpo del artículo.

NOTA: Los autores se someten al proceso de selección y arbitraje de la revista Investigaciones Andina. 